\title{
Kleinkindbetreuung und das Recht von Müttern auf Erwerbsarbeit
}

Neues Denken im krisengeschüttelten Europa*

Seit Mitte der 60er Jahre ist der Anteil erwerbstätiger Frauen in allen europäischen Ländern gestiegen. Die Frage der Kleinkindbetreuung ließ sich deshalb nicht länger beiseite schieben. Dabei bestand die größte Herausforderung für die europäischen Länder darin, das Recht der Mütter auf Erwerbsarbeit und das Recht der Kinder auf angemessene Betreuungseinrichtungen zu verbinden.

Unsere Studie zur Betreuung von Kindern unter drei Jahren bezieht sich auf Belgien, Frankreich, Italien, Schweden und die Europäische Union. Jedes Land hat im wesentlichen zwei Typen von Regelungen entwickelt. Der eine Typus besteht in Erziehungsurlaub und Kindergeld, wodurch es Eltern ermöglicht wird, ihre Berufstätigkeit vorübergehend zu unterbrechen und ihre Kinder selber aufzuziehen. Der andere Typus besteht in kollektiven oder individuellen, öffentlichen oder privaten Betreuungseinrichtungen für Kinder, deren Eltern weiterhin dem Arbeitsmarkt zur Verfügung stehen. Die zur Europäischen Union (EU) gewordene Europäische Gemeinschaft (EG) hat die Mitgliedsstaaten seit den 70er Jahren ermutigt, geeignete Betreuungsarten zu entwickeln, damit die Bestimmungen zur Chancengleichheit von Mann und Frau, die in den Römischen Verträgen festgelegt wurden, eingehalten werden können.

Wir stellen gewisse Übereinstimmungen in der Entwicklung einer neuen Betreuungspolitik fest, wie sie sich seit den 80er Jahren herausgebildet hat. Jenseits unterschiedlicher nationaler Wege treten Tendenzen zu Tage, die im großen und ganzen den gleichen Regeln folgen, welche sich unter den folgenden fünf Schlagworten zusammenfassen lassen: Einschränkung der öffentlichen Ausgaben, Dezentralisierung, Individualisierung, Diversifizierung und Flexibilität.

\section{Neue Herausforderungen, neue Formen von Politik}

Die Politik der Kleinkindbetreuung wurde zwischen den 60er und den 90er Jahren durch neue demographische und ökonomische Entwicklungen mit neuen Herausforderungen konfrontiert. Als der baby boom vom baby crack 
abgelöst wurde, wich die Vollbeschäftigung einer »alltäglich gewordenen« Massenarbeitslosigkeit. Trotz zunehmender »Unterbeschäftigung « konnten sich jedoch die Frauen, insbesondere Mütter von kleinen Kindern, auf dem Arbeitsmarkt behaupten.

So ist der Anteil erwerbstätiger Frauen, insbesondere von Müttern - in Schweden am höchsten, wobei allerdings viele in Teilzeit arbeiten (DauneRichard 1993). Die Arbeitslosenrate von Frauen ist hier sehr gering, geringer als die von Männern. Die Kinderzahl liegt jedoch über dem europäischen Durchschnitt. Wenn es schwedischen Frauen gelingt, bei der höchsten Erwerbsquote auch die meisten Kinder zu bekommen, so liegt das, wie wir zeigen werden, daran, daß ihr Land eine sehr geschickte Politik zur Vereinbarkeit von Beruf und Kinderbetreuung entwickelt hat.

Auch in Belgien und Frankreich ist der Anteil weiblicher Erwerbstätiger sehr hoch, und die Geburtenrate liegt (trotz sinkender Tendenz) ebenfalls über dem europäischen Durchschnitt. Diese beiden Länder haben traditionsgemäß großzügig ausgestattete Betreuungseinrichtungen für Kleinkinder. Zweifellos belasten familiäre Verpflichtungen deshalb das Erwerbsverhalten von Frauen heute weitaus weniger, als dies in der Vergangenheit der Fall war (die Unterbrechung der Erwerbstätigkeit setzt nicht mehr, wie noch in den 60er Jahren, mit dem ersten Kind ein, sondern erst nach dem dritten).

Die Italienerinnen hingegen haben die wenigsten Kinder und sind auch im geringsten Umfang erwerbstätig (die Erwerbsbevölkerung umfaßt nur wenig mehr als ein Drittel Frauen). Weibliche Teilzeitarbeit ist kaum entwickelt. Die Vereinbarkeit von Beruf und Familie regelt sich in Italien eher durch Strategien zur Verringerung der Familiengröße.

Die 80er und 90er Jahre zeichnen sich auch durch eine Bündelung und Neudefinition staatlichen Handelns aus. Es werden nicht mehr universelle, d.h. für alle gleiche Leistungen angestrebt, sondern eine auf Personengruppen orientierte Kleinkindbetreuung. Auch verlieren die Nationalstaaten im Rahmen der EU gewissermaßen an politischem Gewicht. Die Kontrolle staatlicher Wirtschafts- und Sozialpolitik verlagert sich seither: von unten nach oben, hin zur Europäischen Union, und von oben nach unten, hin zu den örtlichen Behörden. Auf allen Ebenen entwickelt sich ein Streben nach mehr Demokratie und nach regionaler Autonomie, was teilweise durch die zunehmende Rekonfiguration der Machtapparate und die Verlagerung von Entscheidungszentren auf infranationale Autoritäten erklärt werden kann.

Die Zeichen der Zeit stehen nicht mehr auf Vollbeschäftigung, wie noch in den Nachkriegsjahren. Die neuen Politikformen sind zu Instrumentarien geworden, mit denen »Resistenz« und »Hindernisse« gegenüber strukturellen Veränderungen beseitigt werden sollen (Mahon 1991). Der politische Akzent liegt eher auf Effizienz als auf Gleichheit und eher auf den Bedürfnissen der Arbeitgeber als auf den Forderungen der Arbeitnehmer. Auch die Familienpolitik soll die Kosten der Wirtschaftskrise mittragen. Überall, außer in Schweden, sinkt der Anteil des Bruttoinlandsprodukts (BIP), der für Familienleistungen ausgegeben wird. Zwar sind die Kosten für die soziale Sicherung seit 1980 kontinuierlich angestiegen, aber die Ausgaben für den Bereich »Familie/Mut- 
terschaft « haben sich zugunsten anderer Bereiche verringert, insbesondere zugunsten der Alterssicherung und der Arbeitslosenversicherung. Gleichzeitig ist die Bevölkerungskrise spürbar geworden. Die Aussicht, daß eine immer kleiner werdende Schicht Erwerbstätiger eine wachsende Rentnerpopulation »ernähren « wird, hat die politischen Entscheidungsträger in Schrecken versetzt. Die demographische Entwicklung ist somit zu einer Frage der Sozialpolitik geworden, während Familienpolitik sich stark zu einer Politik zwischen den Generationen gewandelt hat (Dumon 1996, 31-32).

Unter diesen Voraussetzungen mußte die Politik zur Betreuung von Kleinkindern neu überdacht werden. Die Nationalstaaten und die Gebietskörperschaften standen vor einem doppelten Paradox. Erstens: Obwohl die Zahl von Kindern unter drei Jahren abnimmt (außer in Schweden), gibt es durch die steigende Erwerbstätigkeit von Müttern einen wachsenden gesellschaftlichen »Bedarf« an Betreuungseinrichtungen. Zweitens: Die starke Nachfrage nach Infrastrukturen zur Kinderbetreuung tritt in Zeiten rigider Haushaltsplanung und einer Umstrukturierung des Arbeitsmarktes auf. Die politischen Akteure standen bei der Konturierung neuer Politikformen häufig vor der Aufgabe, entweder Arbeitsplätze wieder frei zu machen (z.B. durch die Einführung von Erziehungsurlaub) oder neue zu schaffen (durch finanzielle Subventionen für Eltern, die Tagesmütter oder Krippen in Anspruch nehmen). Familienpolitik ist so in vielen Fällen zu einer Art Ersatzrad für die gescheiterte Beschäftigungspolitik geworden.

\section{Modelle sozialer Geschlechterbeziehungen: Vorstellungen und ihre Auswirkungen}

Zum Verständnis der Veränderungen, die sich seit der Nachkriegszeit in der Kleinkindbetreuung ergeben haben, sollen im folgenden Vorstellungen von berufstätigen Müttern analysiert werden, wie sie die politischen Akteure geprägt und vermittelt haben (Jenson 1989).

In den vier untersuchten Ländern gab es 1945 einen allgemeinen Konsens darüber, daß für die Kindererziehung am besten die Mütter geeignet seien, auch wenn fortschrittlichere Ideologien ein wenig davon abwichen. Seit den $60 \mathrm{er}$ Jahren, in denen die Zahl weiblicher Erwerbstätiger vor allem im öffentlichen und privaten Dienstleistungssektor stark anstieg, begannen sich die Vorstellungen über die Beziehungen zwischen Männern und Frauen zu verändern. Zunehmend spielte nun auch der in den Römischen Verträgen (1958) konzipierte neue Akteur, die Europäische Gemeinschaft, auf der politischen Bühne eine entscheidende Rolle. Unter dem Einfluß der politischen Linken und des Feminismus setzte sich schließlich in den verschiedenen Staaten der Europäischen Gemeinschaft das Bewußtsein durch, daß zur »Vereinbarkeit von Familie und Beruf « und zur Förderung der Geschlechtergleichheit öffentliche Dienstleistungen nötig seien.

In Schweden wurde das neue Modell der Geschlechterbeziehungen am deutlichsten sichtbar, da dort das Prinzip der Gleichheit von Mann und Frau 
praktiziert wurde. Die Feministinnen der Sozialdemokratischen Partei stellten die Vorstellung von zwei »verschiedenen, aber gleichwertigen « Geschlechtern in Frage, weil sie davon ausgingen, daß Gleichheit durch die Schaffung öffentlicher Kinderbetreuungseinrichtungen erst noch hergestellt werden müsse. Elternurlaub und die zunehmende Einrichtung von Kinderkrippen, auf kommunaler und privater Ebene, gehen auf den Beginn der 70er Jahre zurück. In Frankreich beriefen sich die linken Kräfte auf die Moderne und auf den republikanischen Gleichheitsdiskurs, um die Schaffung von Betreuungseinrichtungen voranzubringen (Jenson/Sineau 1995). Während der Kampagne zur Präsidentschaftswahl von 1974 versprachen sowohl François Mitterand, der Kandidat der vereinigten Linken, als auch Valéry Giscard d'Estaing, der Vertreter der bürgerlichen Mitte, die Anzahl der Krippenplätze stark zu erhöhen. In Belgien traten in den 70er Jahren vermehrt Frauen mit Forderungen nach der Vergesellschaftung der Kleinkindbetreuung auf, die sie als conditio sine qua non ihrer tatsächlichen Gleichberechtigung definierten. Als möglich erschienen verschiedene Veränderungen, eine einheitliche Auslegung der Forderungen ergab sich nicht (Devillé et al. 1995). Um den Gleichberechtigungsansprüchen nachzukommen, wurden einerseits den Kindergärten angeschlossene Krippen gefördert, und es wurde ein Fonds d'équipements et de services collectifs (FESC), ein Sonderetat für öffentliche Dienstleistungen, eingerichtet. Andererseits machten Katholiken und Traditionalisten sich für die Anerkennung der sozialpädagogischen Rolle der Mutter stark und forderten Maßnahmen, die es dieser erlauben sollten, sich selbst um die Kindererziehung zu kümmern. Es wurde die Allocation sociopédagogique (ASP) geschaffen, eine Art Kindergeld.

Auch die Europäische Gemeinschaft begann über die Kommission in diesem Bereich zu intervenieren. Unter Berufung auf Artikel 119 (der die Lohngleichheit garantiert), begann die Kommission Betreuungsdienste zu fördern, damit Frauen ihrer Doppelrolle als Mutter und Erwerbstätige gerecht werden konnten. Ausgehend von Entscheidungen des Europäischen Gerichtshofes und den Richtlinien zur Chancengleichheit aus den 70er Jahren ließ sich die institutionelle Handlungsgrundlage der Kommission durch die Schaffung neuer Organe und Netzwerke schließlich konsolidieren (Ross 1995).

Nur in Italien wurde das Thema der Gleichberechtigung etwas verhaltener angesprochen und man vermied es, Kinderbetreuung und Erwerbstätigkeit der Mütter zu verknüpfen. Hier standen während des Anpassungsprozesses andere Probleme im Vordergrund. Obwohl das Konfliktpotential des Themas »Familie« Rechte und Linke immer wieder zu entzweien vermag, gelang es im Zuge der von Christdemokraten und Linken gleichermaßen angestrebten Modernisierung mit dem Subsidiaritätsprinzip auf Grundlage des familialistischen Modells zu brechen. Um die Suprematie des privaten, und das heißt, kirchlichen Systems zu schwächen, wurden öffentliche Kindergärten eingerichtet. Diese Reform, die 1968 eingeführt wurde, markiert eine wichtige Etappe: Sie stellt das Monopol der Familie in der Kinderbetreuung in Frage.

Die in den 70er Jahren in den vier Ländern geschaffenen öffentlichen Einrichtungen zur Kleinkindbetreuung spiegeln zwei zentrale Auffassungen zur 
Erwerbstätigkeit von Müttern wider. Die erste, die allen einleuchtete, ist, daß die Integration der Frauen in den Arbeitsmarkt zur Verwirklichung der Gleichberechtigung beiträgt. Wenn geeignete Betreuungsdienste zur Verfügung stehen, können Frauen, ähnlich wie Männer, einer Berufstätigkeit nachgehen. Die zweite Idee war weitaus umstrittener. Die einen meinten, daß die Vereinbarkeit beruflicher und familiärer Aufgaben vorrangig eine Frauenangelegenheit sei, auch wenn der Staat die Schaffung von Kindergärten unterstütze. Andere bestritten diese Alleinzuständigkeit der Frauen und wollten im Gegenteil die Väter dazu ermutigen, sich mit ihren Kindern zu beschäftigen. In ihren Augen war es Aufgabe der Gemeinschaft, geeignete Maßnahmen zu entwickeln, damit die Fürsorge für Kleinkinder zwischen Männern und Frauen geteilt werden könne.

\section{Das Auftauchen geschlechter-ungleicher Vorstellungen in der Krise}

Unter dem Einfluß verschiedener Faktoren wurde die zuvor entwickelte Sozialpolitik seit dem Ende der 70er Jahre wieder in Frage gestellt. Politische Umstrukturierungen führten in den verschiedenen Staaten dazu, Garantien zugunsten einer für alle gleichen Staatsbürgerschaft im Namen neuer Werte teilweise wieder aufzugeben. In Belgien und Italien, wo die Verfassung regionale Entscheidungsebenen vorsieht, hat sich die Politik am radikalsten von traditionellen nationalen Normen entfernt. Und selbst in Frankreich und Schweden, Ländern mit einer Verwaltungseinheit, dominiert das Thema der Dezentralisierung gegenüber der Reduktion regionaler Unterschiede und der sich daraus entwickelnden sozialen Ungleichheiten.

Zum Teil haben die Nationalstaaten auch die Idee aufgegeben, die den Kern staatsbürgerlicher Regime der Nachkriegszeit bildete, nämlich Gleichheit vermittels Sozialausgaben zu fördern. Wenngleich die Politik in Italien sich Haushaltskürzungen und einer Reduktion des Staatsapparates lange widersetzen konnte, hat diese in letzter Zeit doch an Boden verloren. In Frankreich ist es den neoliberalen Kräften seit 1983 gelungen, die Politik der sozialistischen Regierungen neu zu orientieren, die in den beiden siebenjährigen Amtszeiten von François Mitterand ihr Programm zur Wiederankurbelung der Wirtschaft zugunsten einer strengen Sparpolitik aufgegeben hat. In Schweden hat die Rückkehr der Sozialdemokraten an die Macht weder 1982 noch 1994 die Veränderungen, welche die bürgerlichen Regierungen vorgenommen hatten, wieder rückgängig machen können. In Belgien hat sich die Rhetorik der Haushaltszwänge auf allen Ebenen der Regierung spürbar durchgesetzt. Gleichzeitig haben die Finanzprobleme der Kommunen einen tiefen Graben zwischen dem »Recht « auf bestimmte Leistungen und ihrer tatsächlichen Verfügbarkeit aufgerissen. Letztendlich hat die Europäische Union in hohem Maße selber zu dieser Entwicklung beigetragen. Der gemeinsame Markt, Konvergenzkriterien und die Perspektive der Währungsunion dienten als wirksame Disziplinierungsmechanismen.

Zwar wurde das egalitäre Modell der Mann-Frau-Beziehung in allen unter- 
suchten Ländern in Frage gestellt, aber weder waren die Form noch das Aus$\mathrm{maß}$ dieser Infragestellung überall die gleichen. Die Europäische Union, die immer bemüht war, das Konzept der Vereinbarkeit von Erwerbsarbeit und Kinderbetreuung auf beide Elternteile auszudehnen, hat unter unseren fünf Länderbeispielen zusammen mit Schweden die am stärksten egalitär ausgerichtete Perspektive. Die Politik der Chancengleichheit der Kommission, für die das Réseau sur les modes de garde d'enfants zuständig ist, hat ihre progressive Sichtweise in der Verbindung von Arbeit und Betreuung während der 80er und 90er Jahre beibehalten. Ihre Bemühungen sind allerdings verpufft, da die Kommission selber in den letzten Jahren zum Rückzug gezwungen wurde.

In Schweden ist die Hegemonie des egalitären Geschlechtermodells offenbar erst seit kurzem bedroht. Gegenwärtig propagiert eine sozialdemokratische Regierung die Ausweitung von privaten Diensten zur Kleinkindbetreuung. Der schwedische Egalitarismus, einst eine rühmliche Ausnahme, ist als möglicherweise im Schwinden begriffen. Die Anhänglichkeit Frankreichs und Belgiens an das egalitäre Modell der Mann-Frau-Beziehungen ist schon immer recht doppeldeutig gewesen, auch bereits in den 70er Jahren. Traditionelle Auffassungen koexistierten mit solchen, die durch die Arbeitsmarktintegration der Frauen Geschlechtergleichheit fördern wollten. In Belgien ist diese Koexistenz am sichtbarsten. Auch wenn die Traditionalisten in Frankreich im Laufe der 70er Jahre zu einer unzufriedenen Minderheitengruppe verkümmerten, werden sie doch niemals völlig verschwinden. Als das Nachkriegsmodell schwächer wurde, traten sie aus dem Schatten heraus und verlangten nach Maßnahmen, die an einer traditionellen Aufgabenteilung zwischen den Geschlechtern festhielten. Allerdings konnten sich die Traditionalisten niemals vollständig durchsetzen, und das Recht der Frauen auf Erwerbsarbeit wurde niemals bestritten.

\section{$Z u$ einer neuen Aufteilung der Sozialzeit?}

Während die Regierungen Maßnahmen zur besseren Vereinbarkeit von Erwerbsarbeit und Familie entwickelten, stießen sie auf das Problem der Verfügbarkeit über die soziale Zeit. In Schweden wurden Eltern seit Beginn der 70er Jahre ermutigt, ihre Arbeitszeiten zu überdenken, um berufliche und familiäre Pflichten vereinbaren zu können. Elternurlaub und das Recht auf Teilzeitarbeit ermöglichten es den Eltern, ihre Arbeitszeiten zu verkürzen. Trotz des Widerstands von Feministinnen, die eine andere Zeiteinteilung, nämlich einen 6Stunden-Arbeitstag für alle, bevorzugt hätten, erlauben die in Schweden ergriffenen Maßnahmen den Eltern, die Betreuung ihrer Kleinkinder selbst zu übernehmen. Der staatliche Diskurs wendet sich an beide Elternteile, und es wurden konkrete Anstrengungen unternommen, um die Väter zu ermutigen (später zu zwingen), ebenfalls Elternurlaub zu nehmen. Diese glückliche Lösung des Dilemmas Arbeitszeit versus Familienzeit wird vielleicht vor allem deshalb keinen Bestand haben, weil die Verknappung der Erwerbsarbeit unterschiedliche Formen von Elternurlaub möglicherweise schwieriger macht. Es 
ist aber noch zu früh, um langfristig Konsequenzen des Niedergangs des schwedischen Modells für die Vorstellungen über erwerbstätige Mütter zu analysieren.

In Frankreich und Belgien hat sich der Staat entschieden, den Forderungen der Unternehmer nach flexibleren Arbeitskräften nachzugeben. Mütter von Kleinkindern stellen nun ein interessantes Reservoir von bezahlten Teilzeitkräften dar, und sie sind gute Anwärterinnen für Berufsunterbrechungen (z.B. durch Erziehungsurlaub und Kindergeld in Frankreich; freiwillige Unterbrechung der Erwerbstätigkeit in Belgien). Während der Elternurlaub und entsprechende Lohnersatzleistungen in Schweden »universell« sind, läßt sich das von Belgien und Frankreich nicht behaupten. Niedrigere Löhne oder Gehälter, negative Konsequenzen für Frauen, die sich nach dem Elternurlaub wieder in den Arbeitsmarkt integrieren wollen, all das sind Faktoren, die die These stiutzen, daß sich diese Leistungen an gering entlohnte und wenig qualifizierte Arbeitskräfte richten (Afsa 1996). Bei der Konzeption dieser Leistungen waren in beiden Ländern gleichheitsfördernde oder feministische Argumente (im Unterschied zu Schweden) nicht im mindesten maßgeblich. Das bedeutet, daß bestimmte Gruppen erwerbstätiger Frauen nicht mehr dieselben Voraussetzungen wie die Männer haben, zumindest wenn sie jung sind. Es tut sich eine Kluft zwischen Männern und Frauen in der Frage auf, wie sie Arbeitszeit und Familienzeit verbinden können.

Nicht alle erwerbstätigen Mütter in Frankreich und Belgien sind jedoch bereit, derartige Formen der Vereinbarkeit zu akzeptieren. Denjenigen Frauen, die, wie ihre männlichen Kollegen, einer qualifizierten und gut bezahlten Vollzeitbeschäftigung nachgehen wollen, stehen andere Optionen offen. Gelingt es ihnen nicht, einen der seltenen Krippenplätze »zu ergattern«, können sie ein »Kindermädchen « oder eine Haushaltshilfe einstellen, dafür gibt es verschiedene Zuschüsse oder Steuererleichterungen. In Belgien und Frankreich lassen sich die Frauen also in zwei Kategorien teilen. Vorstellungen über erwerbstätige Mütter unterscheiden sich je nach ihrer sozialen Klasse.

Italien geht eigene Wege. Widersprüchliche Vorstellungen von der Berufstätigkeit der Mütter und von den Mann-Frau-Beziehungen koexistieren und sind aufgrund der in Italien lange vorherrschenden politischen Unbeweglichkeit intakt geblieben. Die politische Klasse Italiens ist die einzige, die niemals eine klare Verbindung zwischen Geschlechtergleichheit und Kleinkindbetreuung hergestellt hat. In diesem Land existiert die stärkste Ausprägung von privatisierten Betreuungssystemen. Eltern, die sich für Kinder entscheiden, müssen auf sich allein gestellt eine Lösung für die Vereinbarung von Erwerbsarbeitszeit und Familienzeit finden. Sie können sich entweder an ihre Familie wenden oder private Betreuungsdienste in Anspruch nehmen. Eine der Lösungen, die zahlreiche junge italienische Familien wählen, ist ebenfalls eine »private «: sie besteht darin, kein Kind oder nur ein einziges zu bekommen, und bis zum Schuleintritt an einer Betreuungslösung zu »basteln«. 


\section{Verschiedene Modalitäten von Betreuung}

Der Vergleich zwischen den vier Ländern zeigt eine große Ungleichheit im Zugang zu Betreuungsdiensten, die von der öffentlichen Hand finanziert werden. Der relative Reichtum an öffentlicher Betreuung in Schweden (33\% in der Altersklasse von null bis drei Jahren), in Belgien (30\%) und in Frankreich (23\%) kontrastiert mit einem ärmlichen Angebot in Italien (6\%). Zu den nationalen Unterschieden kommen regionale oder lokale Ungleichheiten innerhalb der einzelnen Staaten hinzu. Die Ursache dafür ist die Abhängigkeit der Betreuungsdienste von lokalen oder regionalen Behörden, deren Wille oder Fähigkeit, diese Dienste zu finanzieren, sehr variabel ist.

Frankreich und Italien sind zwei entgegengesetzte Beispiele. Frankreich bietet die breiteste Palette von öffentlichen Betreuungsarten, während sich Italien durch ein mageres Angebot auszeichnet. Da die Kindergärten in Italien nur für Kinder ab drei Jahren zugänglich sind, stellen die asili nido (Zentren für Kinder von 3 bis 36 Monaten) praktisch die einzige Möglichkeit dar, kleine Kinder aufzunehmen. In den letzten zehn Jahren haben die lokalen Behörden, vornehmlich in Nord- und Mittelitalien, neue Dienste entwickelt, die nuove tipologie, mit gleitenden Öffnungszeiten und einer Teilzeitbetreuung. In Frankreich dagegen ist eine große Vielzahl vorhanden. Das Erziehungssystem bietet eine wachsende Anzahl von Kindergartenplätzen ab dem zweiten Lebensjahr an. Das französische Modell zeichnet sich durch die herausragende Rolle aus, die das Erziehungssystem bei der Kleinkindbetreuung spielt. Die Kindergärten umfassen mehr als $56 \%$ der im öffentlichen Bereich verfügbaren Plätze. Das Sozialsystem bietet drei Strukturtypen an: öffentliche Krippen $(26, \%$ der Plätze), private Krippen (14,7\% der Plätze) und schließlich Kinderhorte für kurzzeitige Betreuung. Belgien steht dem französischen Modell in zweierlei Hinsicht sehr nahe. Einerseits bietet es eine große Vielfalt von Diensten an (Kindergärten, Betreuungszentren und zeitweilige Betreuung durch Kindergärtnerinnen), andererseits ist das Gewicht der im Schulsystem verankerten Kindergärten gegenüber den Fürsorgedienstleistungen (Betreuungzentren und zeitweilige Betreuung), sehr groß. Schweden schlägt schließlich ein eigenes Modell vor. Die Auswahlmöglichkeiten unter den öffentlichen Betreuungsdiensten sind sehr gering, vor allem deshalb, weil das Bildungssystem keine Rolle bei der Betreuung von Kindern unter drei Jahren spielt. Dennoch beeinflussen die beschränkten Auswahlmöglichkeiten den Gesamtumfang der Dienste nicht. Fast ein Drittel der Kinder unter drei Jahren findet einen Platz.

Welche Betreuungswege auch eingeschlagen werden, alle sind im Rahmen aktueller Sparmaßnahmen beträchtlichen finanziellen Zwängen unterworfen. Überall gibt es verringerte staatliche Zuschüsse und eine spürbar zunehmende Belastung der Gemeinden. Auch läßt sich beobachten, daß der Elternbeitrag zur Finanzierung der unterschiedlichen öffentlichen Betreuungsdienste zwar von Land zu Land verschieden, aber dennoch überall stark gestiegen sind. Paradoxerweise sind die Betreuungskosten in den beiden sonst so gegensätzlichen Ländern Italien und Schweden am geringsten.

In den vier Länderbeispielen haben die Betreuungsdienste einen redistribu- 
tiven Charakter, d.h. die Eltern beteiligen sich an den Kosten je nach ihrem Einkommen und nach der Kinderzahl. In Belgien und Frankreich führen Steuersenkungen allerdings zu einer Verringerung der Redistributionseffekte. Das System begünstigt Familien, die höhere Steuern zahlen, die »Wohlhabenden « gehören. In Italien und Schweden sind steuerliche Vergünstigungen dagegen unbekannt.

\section{Individuelle, elterliche und andere Betreuungsarten}

Das Neue des letzten Jahrzehnts ist die Vielfältigkeit in der individuellen Betreuung, die durch die Einführung des Erziehungsurlaubs entstanden ist. Schweden hat hier eine Vorreiterrolle gespielt, da es den Erziehungsurlaub schon 1974 einführte. Frankreich und Italien zogen 1977 nach. Belgien hat erst 1985 eine interruption volontaire de carrière (IVC), als ein freiwilliges Aussetzen oder eine »Pause in der Berufslaufbahn « eingeführt.

Die Ausgestaltung des »Urlaubsgelds « sagt einiges über die Konzeption des Erziehungsurlaubs aus. Es gibt zwei unterschiedliche Modelle: Das Urlaubsgeld wird entweder als Pauschalbetrag (von bescheidener Höhe) oder als Prozentsatz des vorherigen Gehalts gezahlt. In Frankreich und Belgien ist das erste Modell vorherrschend, die Pauschalzahlung. In Frankreich beträgt das aktuelle Erziehungsgeld gut die Hälfte des dynamisch angepaßten Mindestlohns (SMIC). In Belgien bekommen alle Erwerbstätigen, die eine IVC in Anspruch nehmen, einen bescheidenen Pauschalbetrag, der der Untergrenze des Arbeitslosengeldes entspricht. In Italien und Schweden entspricht das Erziehungsgeld einem bestimmten Prozentsatz des Gehalts, der allerdings jeweils sehr verschieden ausfällt. In Italien werden im allgemeinen $30 \%$ des $\mathrm{Ge}$ halts gewährt. Viele Frauen im öffentlichen Sektor erhalten allerdings einen tarifvertraglich abgesicherten höheren Prozentsatz. In Schweden gibt es während des Erziehungsurlaubs, der beiden Elternteilen zusteht, einen weit höheren Betrag, der gegenwärtig $80 \%$ des letzten Gehalts entspricht.

Das schwedische Erziehungsurlaubsgesetz entspricht der Versicherungssystematik und orientiert sich an der »Qualität des/der Erwerbstätigen«. Der Erziehungsurlaub ist überdies mit einem umfassenden Sozialversicherungsschutz und mit einer Arbeitsplatzgarantie ausgestattet. In Frankreich und Belgien ist dies nicht der Fall. So bietet der Erziehungsurlaub in Frankreich eine nur ungenügende Garantie zur Wiedereinstellung und wird nur zu $50 \%$ auf die Rente und auf damit verbundene Vorteile angerechnet. Er knüpft also nicht an eine Erwerbstätigkeit an.

Der Erziehungsurlaub ist in gewissem Sinne flexibel, da er auch in Teilzeit genommen werden kann. In Belgien wird dazu die Einwilligung des Arbeitgebers benötigt. In Frankreich wurde die Möglichkeit, Erziehungsurlaub auf Teilzeit zu nehmen, durch das Familiengesetz vom 25. Juli 1994 erleichtert (Afsa 1996). Das schwedische System ist am geschmeidigsten: die Eltern können einen Teil oder die Gesamtheit ihres bezahlten Urlaubs als Vollzeit, Halb- 
zeit oder Viertelzeit nehmen. Sie können ihren Erziehungsurlaub auf einmal oder in mehreren Etappen nehmen, den Erziehungsurlaub unter sich aufteilen, von Vollzeit- auf Teilzeit und umgekehrt wechseln, und zwar so lange, bis das Kind acht Jahre alt ist. Insgesamt ähnelt der Erziehungsurlaub einem »ZeitKapital«, das den Eltern zur Verfügung steht (Hantrais/Letablier 1995, 38).

Schweden ist das Land, in dem Erziehungsurlaub am häufigsten in Anspruch genommen wird, von 1989 bis 1990 von schätzungsweise fast allen berufstätigen Müttern (98\%). Diese große Inanspruchnahme hat dazu beigetragen, daß sich die Nachfrage nach Krippenplätzen verringerte. In den anderen Ländern ist der »Erfolg « des Erziehungsurlaubs weitaus geringer. Überall wird er hauptsächlich von Frauen in Anspruch genommen. Eine von beiden Geschlechtern gleiche Inanspruchnahme steht noch aus, auch in Schweden, obgleich es dort erklärtes Ziel ist, mit Hilfe des Erziehungsurlaubs und des Erziehungsgeldes, das sich nach der Höhe des individuellen Gehalts bestimmt, die Gleichstellung der Geschlechter zu fördern.

Wenn sich zahlreiche Eltern nun wieder auf dem privaten Markt für Betreuungsdienste umschauen, so ist das nicht immer eine freiwillige, sondern häufig auch eine durch fehlende öffentliche und staatlich subventionierte Betreuungseinrichtungen »erzwungene « Entscheidung. Die Qualität der privaten Betreuungsdienste ist »von den Summen abhängig, die die Eltern aufbringen können « (Réseau 1995, 60) und hat auch mit dem Grad der Professionalität der Kindergärtnerinnen zu tun, die einen Teil der privaten $\mathrm{Be}$ treuungsdienste übernehmen. Es gibt große Unterschiede, von der Kindergärtnerin, die freiberuflich und ohne staatliche Kontrolle tätig ist (wie in Italien) bis zu staatlich geprüften Kindergärtnerinnen, die zwar unabhängig sind, aber kontrolliert werden (wie in Belgien oder Frankreich). Aber selbst in den Ländern, in denen freiberufliche Kindergärtnerinnen Auflagen erfüllen müssen, haben die »Kinderprofis « recht unbefriedigende Arbeitsbedingungen, was das Gehalt, soziale Rechte und Weiterbildungs- und Aufstiegsmöglichkeiten betrifft.

Trotz der notorischen Unzulänglichkeiten der Statistiken über private Betreuungsdienste läßt sich Folgendes feststellen: Schweden weicht von den drei anderen Ländern ab. Es zeichnet sich durch die Schwäche des privaten Betreuungsangebots aus, das nur sieben bis zehn Prozent der Kinder von ein bis sechs Jahren abdeckt. In Belgien umfaßte die individuelle Betreuung durch unabhängige oder stationäre, diplomierte Kindergärtnerinnen 1990 etwa 23\% der Kinder unter drei Jahren in dem flämischen Bevölkerungsanteil und 16\% in dem französischen Bevölkerungsanteil (Dubois/Humblet/Deven 1994). In Frankreich ist der Umfang privater Betreuung größer, sie stellt $60 \%$ der Plätze für Kinder unter drei Jahren zur Verfügung. Staatliche Instanzen unterstützen Eltern (finanziell und über Steuererleichterungen), damit sie private Dienstleistungen von Kindergärtnerinnen in Anspruch nehmen. 1986 wurde die Allocation de garde d'enfants à domicile (AGED) geschaffen, eine finanzielle Unterstützung, die es Haushalten mit höherem Einkommen ermöglicht, mehr als die Hälfte der Kosten für eine Hausangestellte auf die Allgemeinheit abzuwäl- 
zen. 1990 kam eine Familienzulage hinzu, die Aide aux familles pour l'emploi d'une assistante maternelle agrée (AFEAMA), die vor allem den Mittelschichten zugute kam. Durch diese Hilfe wurde es für sie vorteilhafter, ihre Kinder privat von einer Kindergärtnerin hüten zu lassen, anstatt auf kollektive Einrichtungen zurückzugreifen. Es muß jedoch betont werden, daß weder die AGED noch die AFEAMA irgendeinen »sozialen « Charakter haben: diese Leistungen sind keinerlei Einkommensbeschränkungen unterworfen und werden überdies von starken Steuerbegünstigungen für die wohlhabenden Klassen begleitet. ${ }^{1}$

Will man nun unsere vier Länder daraufhin klassifizieren, wie sie das Verhältnis von Familie und Erwerbsarbeit lösen, stellen wir fest, daß Schweden die geschickteste Politik zur Vereinbarkeit beider Bereiche entwickelt hat. Angesichts der neuen Verhältnisse am Ende des 20. Jahrhunderts - eine hohe Frauenerwerbstätigkeit erfordert eine angemessene Politik zur Kinderbetreuung - ist allein dieses Land wirklich dieser Herausforderung gerecht geworden, indem die Vereinbarkeitsproblematik Priorität in der Familienpolitik erlangt hat. Nur hier wurden die politischen und finanziellen Mittel bereitgestellt, um in relativ kurzer Zeit das Problem der Betreuung von Kleinkindern, deren Eltern berufstätig sind, zu lösen, sowohl durch die Schaffung von Krippen - die vom Gesetz als ein »obligatorischer öffentlicher Dienst « definiert wurden - als auch durch einen Erziehungsurlaub, der als eine regelrechte Versicherung gegen das »Kinderrisiko« konzipiert wurde. Solch eine Politik wurde im übrigen immer von dem Bestreben geleitet, die Gleichstellung der Geschlechter zu verwirklichen: die Männer wurden - durch bestimmte Modalitäten des Elternurlaubs - stets dazu angeregt, die Erziehungsverantwortung zu teilen. ${ }^{2}$ Diese Politik wurde übrigens nicht so sehr als Familienpolitik beschrieben, sondern eher als eine »Politik für die Kinder « und als eine »Politik der Geschlechtergleichheit«, »In diesem Modell werden die Kinder als Staatsbürger betrachtet und nicht nur als Konsumenten von Gütern und Dienstleistungen, und als Staatsbürger haben sie Rechte, die Rechte, betreut und erzogen zu werden « (Hantrais/Letablier 1995, 44). Die Wirtschaftskrise, die Schweden viel später als andere europäische Länder erreicht hat, hat an dieser doppelten Dimension der schwedischen Politik nichts geändert. Das beste Zeichen dafür ist, daß die öffentlichen Ausgaben für die Betreuung von Kleinkindern auch in den letzten Jahren kontinuierlich gestiegen sind.

Weder Belgien noch Frankreich haben der öffentlichen Betreuung von Kleinkindern in ihrer Familienpolitik Priorität eingeräumt. Sie bevorzugen den »privaten Weg «. Sie haben es nicht vermocht, Mittel bereit zu stellen, um dem steigenden Bedarf an unterschiedlichen Formen der Kinderbetreuung gerecht zu werden; was um so schwerschwiegender ist, weil - im Gegensatz zu Schweden - Teilzeitarbeit in Frankreich nicht zur Tradition weiblicher Erwerbsarbeit gehört und auch heute nur von geringer Bedeutung ist (mehr als $70 \%$ der Berufstätigen arbeiten Vollzeit). Frankreich weist große Mängel bei der öffentlichen Kleinkindbetreuung auf. Gewiß wurde dort seit den 80er Jahren eine Politik der sogenannten »Diversifizierung der Wahlfreiheit « verfolgt, indem spezielle Dienstleistungen zugunsten der privaten Kleinkindbetreuung 
entwickelt wurden, wie Erziehungsurlaub, Erziehungsbeihilfen und Steuersenkungen für Betreuungskosten.

Doch liegt der hauptsächliche Nachteil einer Politik, die auf finanziellen Unterstuitzungsleistungen beruht darin, daß diese faktisch die Betreuung durch die Mutter begünstigt, und damit auch eine »Orientierung « auf die (berufliche) Chancenungleichheit zwischen Männern und Frauen. Gleichzeitig hat sich die Politik, die auf Sachleistungen ausgerichtet war, wie die Einrichtungen öffentlicher Krippen, als ausgesprochen zurückhaltend erwiesen. Diese Einschätzung teilen auch befragte Eltern, die ein starkes Gefühl der Unzufriedenheit bekunden, das aus »der Unangemessenheit des öffentlichen Hilfsangebots gegenüber den gesellschaftlichen Erwartungen« herrührt (Commaille 1993, 91). Nach einer französischen Umfrage konnten 57\% der Eltern, die sich für eine Krippe entschieden hatten, keinen Krippenplatz finden (Hatchuel 1989).

Das italienische Modell bildet den Gegenpol zum schwedischen Modell. Je mehr die Betreuung kleiner Kinder eine Familienangelegenheit bleibt, desto weniger wird vom Staat erwartet, in diesem Bereich aktiv zu werden. Unter diesen Voraussetzungen belasten Erziehungsaufgaben die Beziehung italienischer Mütter zur ihrer Erwerbstätigkeit schwer. Es ist deshalb nur nachvollziehbar, daß die Erwerbsquote italienischer Mütter in dem Maße sinkt, in dem sich ihre Kinderzahl erhöht. Seit 1945 hat der italienische Staat gezeigt, daß er keinerlei Interesse daran hat, sich - neben den Kindergärten für Dreijährige und Ältere - um die Betreuung von Kleinkindern zu kümmern. Ende des 20. Jahrhunderts werden in Italien die Probleme der Betreuung von Kleinkindern berufstätiger Eltern immer noch vorrangig durch die Solidarität der Familie als durch den Wohlfahrtsstaat gelöst.

Aus dem Französischen von Ronald Voullié.

\section{Anmerkungen}

* In diesem Artikel werden die Ergebnisse einer vergleichenden Studie zusammengefaßt: Jane Jenson/Mariette Sineau dir. Qui doit garder le jeune enfant? Modes d'accueil et travail des mères dans l'Europe en crise. Paris: Librairie

Générale de Droit et de Jurisprudence, 1998.

1 Immerhin sind die steuerlichen Vorteile, die mit der AGED verbunden sind, im Jahre 1998 auf Betreiben von Martine Aubry (damalige Ministerin für Beschäftigung und Solidarität in der Regierung von Lionel Jospin) gesenkt worden.

2 Das Réseau des Modes de garde der Europäischen Kommission ist stark vom schwedischen Erziehungsurlaub beeinflußt worden, als es seinen Beitrag über die Vereinigung von Familie und Arbeit erarbeitete.

\section{Literatur}

Afsa, Eric (1996): L'activité féminine à l'épreuve de l'allocation parentale d'éducation. In: Recherches et Prévisions, Nr. 46, Dezember, S. 1-8. 
Commaille, Jacques (1993): Les stratégies des femmes. Travail, famille et politique. Paris.

Daune-Richard, Anne-Marie (1993): Activité et emploi des femmes: des constructions sociétales différentes en France, an Royaume-Uni et en Suède. In: Sociétés contemporaines, Nr. 16, S. 125-143.

Deville et al. (1995): Belgique. Du jeu des clivages á la politique des compromis. In: L. Assier - Andrien et J. Commaille dir., Politiques des lois en Europe. Paris: LGDJ.

Dubois, Alain/Humblet, Perrine/Deven, Fred (1994): L'accueil des enfants de moins de trois ans. Brïssel, CRISP, coll. »Courrier hebdomadaire«, Nr. 1463-1464.

Dumon, Wilfried (1991): Les politiques nationales des États membres de la Communauté européenne en 1991. Observatoire européen des politiques familiales nationales. Brussel: Commission des Communautés. In: J. Commaille u.F. de Singly (Hrsg.): »La politique familiale « Problèmes politiques et sociaux, Nr. 761. Paris: La Documentation française.

Fagnani, Jeanne (1992): Travail et fécondité en France et en Allemagne de l'Ouest. In: Revue Française des Affaires Sociales, Nr. 2.

Hantrais, Linda/Letablier, Marie-Thérèse (1995): La relation famille-emploi. Une comparaison des modes d'ajustement en Europe. Centre d'Etudes de l'Emploi: Dossier Nr. 6, Nouvelle série.

Hatchuel, Georges (1989): Accueil de la petite enfance et activité féminine. Paris: CREDOC, rapport à la CNAF.

Insee (1992): Les enfants de moins de trois ans. Paris: INSEE.

Jenson, Jane (1989): Paradigms and Political Discourse: Protective Legislation in France and the United States Before 1914. In: Canadian Journal of Political Science, Bd. XXII.

Jenson, Jane/Sineau, Mariette (1995): Mitterrand et les Françaises. Un rendez-vous manqué. Paris. Presses de Sciences Po.

Jenson, Jane/Sineau, Mariette dir. (1998): Qui doit garder le jeune enfant? Modes d'accueil et travail des mères dans l'Europe en crise. Paris. Libraivie Generda de Droit et de Jurisprudence.

Mahon, Rianne (1991): From Solidaristic Wages to Solidaristic Work: A Post Fordist Historic Compromise for Sweden? In: Economic \& Industrial Democracy, 12 (3), S. 295-326.

Maruani, Margaret (1993): Emploi féminin: les mutations en cours. Brussel: Commission des Communautés européennes (Unité pour l'égalité des chances - DG V.B.4), multigraphié.

Norvez, Alain (1990): De la naissance à l'école. Santé, modes de garde et préscolarité dans la France contemporaine. Paris: PUF/INED.

Réseau des modes de garde d'enfants de la Commission européenne,

- (1994): Congés spéciaux accordés aux travailleurs qui ont des enfants. Brüssel: Commission européenne, DGV, multigraphié.

- (1995): L'assistance maternelle en Europe. Brüssel: DGV, multigraphié.

- (1996): Une étude des services pour les jeunes enfants. Brüssel: Commission européenne, DGV, multigraphié.

Ronsin et al. (1997): Démographie et politique. Dijon. Edition Universitaires de Dijon. Ross, George (1995): Jacques Delors and European Integration. Cambridge. Policy Press. 